\title{
Dinero digital: Una opción a la regulación del Banco Central ${ }^{1}$ Digital money:
An option to Central Bank regulation
}

DOI: https://doi.org/10.17981/econcuc.40.1.2019.09

Artículo de investigación. Fecha de recepción: 04/12/2018 Fecha de aceptación: 15/04/2019

\author{
Daniela Alejandra Montaña Rubiano \\ Universidad Colegio Mayor de Cundinamarca. (Bogotá, Colombia) \\ damontana@unicolmayor.edu.co
}

Para citar este artículo:

Montaña, D. (2019). Dinero digital: una opción a la regulación del Banco Central. Económicas CUC, 40(1). 145-156. DOI: http://doi. org/10.17981/econcuc.40.1.2019.09

Resumen

Desde la perspectiva económica convencional se puede evidenciar como el dinero ha tomado una fuerza importante dentro de la ciencia económica, son muchos los autores que pretenden conocer el funcionamiento del mismo y como este puede generar afectaciones en la sociedad; prueba de ello, la escuela austriaca al ofrecer una perspectiva radical de no intervención del Estado, planteando un sistema competitivo de banca libre. Mediante el análisis documental se pretende direccionar este artículo hacia la posibilidad de establecer una moneda de carácter privado, desde los planteamientos de la escuela austriaca, analizando el tema con el desafío de plantear el desarrollo de las monedas digitales, y con esto se pretende evaluar la operatividad de las mismas basados en la teoría económica y los beneficios que estas monedas en su conjunto generarían en la economía comparandolas con el sistema monetario regulado por la banca central. Como resultado se logra identificar como en cuanto a las proyecciones de las criptomonedas se muestran complicaciones presentes en la moneda. Y a partir de estas convenciones podemos concluir como el BITCOIN, si es una materialización de la idea austriaca, al cumplir con todos los aspectos mencionados por Hayek y la escuela austriaca, además de tener en manera implícita su forma de competencia con las demás monedas de carácter digital y con el papel moneda.

Palabras clave: Desregulación; monedas digitales; criptografía; libertad bancaria; cadena de bloques

\begin{abstract}
From the conventional economic perspective it can be seen how money has taken an important force within economic science, there are many authors who claim to know how it works and how it can generate effects in society; proof of this, the Austrian school by offering a radical perspective of non-intervention of the State, proposing a competitive system of free banking. By means of documentary analysis, the aim is to direct this article towards the possibility of establishing a private currency, from the approaches of the Austrian school, analysing the subject with the challenge of raising the development of digital currencies, and with this it is intended to evaluate their operability based on economic theory and the benefits that these currencies as a whole would generate in the economy by comparing them with the monetary system regulated by the central bank. As a result, it is possible to identify how, in terms of cryptocurrency projections, complications present in the currency are shown. And from these conventions we can conclude as BITCOIN, if it is a materialization of the Austrian idea, by complying with all the aspects mentioned by Hayek and the Austrian school, in addition to having implicitly its form of competition with the other digital coins and with paper money.
\end{abstract}

Keywords: Deregulation; digital currencies; cryptography; banking freedom; chain of blocks

\footnotetext{
${ }^{1}$ Este artículo es resultado de investigación enmarcado dentro del Semillero de Investigación Agorá Internacional en el pregrado de Economía de la Universidad Colegio Mayor de Cundinamarca(Colombia
} 


\section{INTRODUCCIÓN}

Dentro de la perspectiva económica se puede realizar un análisis de la preponderancia que tiene el dinero dentro del sistema social, viendo en pleno siglo XXI el papel moneda como medio de pago, y al ser originario del siglo XIX, ha perdurado en el tiempo, ya sea por sus aspectos de confiabilidad impuesta o por su característica de "buen" método de intercambio. Sin embargo, se puede realizar una distinción o clasificación del dinero, entre los cuales se trataran: dinero de curso legal (papel moneda) y dinero bancario (tarjetas de crédito, cheques, débito, etc.), no obstante, existe una nueva forma de transar, los denominados medios no convencionales, nombrados de esta manera al no hacer parte de un acuerdo o convenio como los antes mencionados, es decir, no son reconocidos como medio de transacción; estas son las criptomonedas, una combinación de procesos tecnológicos seguros pero con un nuevo enfoque de construcción, estas innovaciones muestran como es posible que la informática de las redes y la criptografía ${ }^{2}$ co-ayuden, y así esta primera instancia tecnológica y motor de consenso sin confianza transforme la tecnología de la contabilidad (Davidson, De Fillipi \& Potts, 2018).

Dicho de otro modo, Davidson et al (2018) dan a conocer la tecnología de las criptomonedas como primera instancia tecnológica por ser un primer acercamiento para remodelar el sistema contable (sobre los libros contables u operaciones en la red), identifican la tecnología detrás de la moneda virtual como la real innovación en el proceso, puesto que la criptografía y el

${ }^{2}$ Técnica de escribir con procedimientos o claves secretas o de un modo enigmático, de tal forma que lo escrito solamente sea accesible para quien sepa descifrarlo blockchain son los reales generadores de la red libre y segura en términos transaccionales para todos los usuarios.

Se pretende caracterizar las criptomonedas como la forma operativa de la propuesta teórica de la escuela austríaca, adicionalmente se cuestiona el origen del monopolio actual de emisión en las monedas, situación que logra generar incertidumbre en otras monedas que tienen posibilidades de competencia con el papel de curso legal y cómo esto ha limitado opciones como el "free banking". La investigación se realiza en aras de conocer si ¿Es el BITCOIN una posibilidad de materializar el sueño austriaco de la banca libre?, mediante un análisis teórico y documental de la escuela austriaca.

Se dividirá el documento en cinco secciones, la primera conocida como introducción y metodología, seguido de teoría austriaca del dinero, donde se tratarán tres puntos importantes para proponer la posibilidad de un sistema en el cuál se de la competencia de monedas más específicamente con las criptomonedas o dinero digital y el dinero de curso legal, lo cual implica analizar: a) la desregulación del dinero, b) los aspectos de la neutralidad del dinero, c) la eliminación del poder de emisión por parte de los Bancos Centrales. Una tercera sección denominada caracterización de las monedas virtuales, dividida en d) blockchain y criptografía y e) condiciones para su aceptabilidad. Por último, se establecerán las conclusiones a las cuales se llegó tras el recorrido de la investigación.

\section{Metodología}

En la realización de esta investigación fue utilizado el análisis documental o enfoque narrativo fijándose en los textos de las escuelas monetaristas y austriacas den- 
tro del debate de la regulación del banco central. Se toman textos de finales del siglo XX hasta la actualidad, permitiendo conocer a fondo las posiciones de las escuelas y a su vez posibilitando crear una idea clara de cada perspectiva. En el transcurso y realización del presente documento se utilizó un enfoque metodológico basado en documentos oficiales como fuente de información, contrastando la realidad a través de un enfoque interpretativo, en una investigación de carácter cualitativo estudiando las características y las cualidades del fenómeno de desregulación.

Teoría austriaca del dinero

Todas las visiones o posturas de las escuelas económicas a lo largo de la historia van en busca del bienestar de las economías y por ende su abanderado es generar una estabilidad; para la escuela austriaca, el problema más grande que tienen los Estados es la regulación del dinero por parte del mismo Estado, generando desequilibrio e impactando fuertemente a las economías. Un claro ejemplo es la reciente crisis económica que ha puesto en duda la regulación bancaria en el año 2008, cuando se dio origen a la burbuja financiera ${ }^{3}$.

Es puesta en duda la función del Estado, debido a la misma estructura del sistema, el cual se ha caracterizado por desequilibrios y debilidades en los sistemas financieros y bancarios, donde la mayor problemática radica en los fallos de intercomunicación que ambos tienen (Estado y sistema financiero). Es claro como esta crisis ha sido una de las más potentes que ha vivido la economía mundial al afectar

\footnotetext{
${ }^{3}$ El Estado fue el promotor de la crisis por un fallo en la regulación de los mercados, ya instaurada la crisis su labor adicional fue la de inyectar dinero de manera extraordinaria, cerca de USD $\$ 1$ billón, para buscar nuevamente la reactivación de las entidades bancarias y evitar la quiebra de muchas otras, como ya venía ocurriendo desde meses atrás.
}

a varias naciones, sin importar la posición geográfica, y esto se debió claramente al nivel de globalización.

Dentro del proceso de crisis puede incluirse un tema posterior de neutralidad, teniendo como ente regulador al Estado el cual debe proporcionar dinero que será convertido en crédito y bajo la coyuntura por la que pasa la economía, el dinero bajo la forma del crédito no puede cumplir su papel de recolector de información, sino que transmitirá falsos mensajes. Para los austriacos, la crisis económica será la consecuencia del desorden causado por la manipulación monetaria, particularmente por la creación de dinero, a través de la intermediación del crédito. Por lo anterior, cabe mencionar como los problemas de la banca experimentados en las crisis económicas son un punto a favor de la visión de la teoría austriaca para permitir dentro de las economías competir y permitir la desregulación del dinero.

\section{Desregulación del dinero}

Al realizar un revisión histórica sobre los métodos de intercambio y transacción por defecto de cada época, se puede evidenciar como este ha atravesado un gran número de transformaciones, pasando por todas las épocas históricas. donde se diversifica la forma más común de intercambio hasta llegar al papel moneda, instaurado a mediados del siglo XIX, y convirtiendose en uno de los pilares de poder que el Estado mas ha monopolizado y usado a su favor, al ser la forma más clara de confianza impuesta en los subordinados de las naciones (Hayek, 1976).

Lo que permitió al papel moneda instaurarse fue la política de libre comercio basada en el concepto de competitividad de carácter general, a tal punto de desarrollarse de manera innata como un proceso 
de selección por el mismo público, quienes apartaron del mercado las monedas de baja calidad por no existir confianza en su elaboración (Hayek, 1976). Justo en esta época se dio el dilema de la convertibilidad, donde se buscaba ser aceptada en distintos mercados con la finalidad de satisfacer los interés de los usuarios.

La regulación nace como estrategia para que los medios de pago no fueran plagiados, es decir, para evitar la falsificación; esta regulación viene desde la acuñación del S. VI a.c. Es el poder de emisión ejercido por las naciones una forma de garantizar la confianza en el sistema monetario, la función de los gobiernos no era tanto la de "producir" dinero sino la de certificar el peso y la calidad de los materiales utilizados universalmente como dinero (Hayek, 1976). Las monedas con las que se realizan las operaciones económicas han sido impuestas por cada nación a pesar de existir otros medios de pago, es decir, el proceso de confianza y credibilidad de la moneda se da a partir de las garantía dadas por cada Estado.

Actualmente la moneda certificada, que cuenta con la credibilidad y garantía es aquella de curso legal aceptada por cada Estado o gobierno; la ley reconoce un solo tipo de dinero: el emitido por el monopolio de la Banca Central, es decir, el acreedor o ciudadano no se puede rehusar a aceptar como medio de pago, tal y como sucedía en siglos pasados. Desde el pensamiento económico se realizan análisis sobre la teoría propuesta por Hayek (1976) en su libro "La desnacionalización del dinero" la cual nace en aras del mejoramiento de estabilidad, calidad de vida y competencia del mercado.

Las posturas dentro del tema de la regulación pueden ubicarse en tres niveles: 1) el de la nueva macro clásica que le da una importancia mínima al Estado sobre- saliendo el enfoque regulador, incluso en periodo de crisis, 2) el enfoque neokeynesiano, el cual asume mayor intervención proponiendo un enfoque contracíclico en las crisis económicas y 3) el enfoque austriaco al sumir por su parte que las crisis ocurren por la intervención del Estado proponiendo el desarrollo de la banca libre, permitiendo la competencia en el mercado de dinero, siempre y cuando exista un respaldo en la emisión en algo real. Los pensadores de la escuela keynesianas se refieren al tema de la capacidad del Estado de regular las crisis y mitigar los procesos inflacionarios en las economías, temáticas abordadas en el desarrollo de la idea austriaca.

Cabe resaltar como la desregulación presenta sus limitaciones puesto que esta depende de la aceptabilidad dada por las transacciones voluntarias de la sociedad; se convierte entonces en una red descentralizada de confianza que no posee un área de regulación propia, adicionalmente el poder ejercido del gobierno durante muchas décadas por la capacidad del Estado como gestor de la invisibilización de las opciones alternativas. Hayek (1976) mencionó el cambio del sistema monetario no es fácil por tres razones importantes: 1) la preponderancia que tiene la relación de suministro de dinero para el crédito, 2) el largo volumen del gasto estatal, 3) los cambios en el suministro relativo del dinero al ser "mucho más perturbador que los cambios en alguna otra circunstancia que afectan los precios y la producción".

Un detractor de la postura hayekiana por el tema de la coexistencia de monedas y la confianza en el gobierno, es Milton Friedman, es reacio al tema de privatización puesto que esto no podría romper la rígida estructura del complejo autoritario. Sin embargo, no desconoce el potencial de las tecnologías, el mismo reconoce que: 
Creo que Internet va a ser una de las fuerzas principales para reducir el papel del gobierno. Lo único que falta, pero que pronto se desarrollará, es un efectivo electrónico confiable, un método mediante el cual en internet pueda transferirse fondos de A a B, sin que A sepa de B, ni B sepa de A (Friedman, 1999).

La posición tomada dentro del artículo es a favor de la desregulación, apoyar la moción propuesta por la visión austriaca: ¿Por qué?, por qué dentro de la imposición estatal de la moneda, y en la era de globalización que se vive, el papel moneda no puede, ni debe ser el único medio avalado por los gobiernos al ser una forma de limitar las economías y acotar el desarrollo, tanto del avance tecnológico como del económico. La intervención durante largo años no ha dejado muy bien posicionados a los entes gubernamentales: crisis, alta volatilidad, incertidumbre (pese a tener el poder de imponer confianza), hambrunas y demás. La posición estatal como se ve actualmente, fuera de ser un garante de la estabilidad del pueblo, termina siendo un garante de estabilidad propia, en pro de los pocos y no de la generalidad.

\section{Neutralidad del dinero}

Con este apartado se pretende dar a conocer la caracterización, las capacidades y cualidades del dinero, y además de ello, identificar como las acciones estatales por convertir el dinero en un bien neutral genera distorsiones en los mercados, Mises (1949) describe como "la moneda constituye factor engendrador de cambios, no porque "circula", sino en razón a que se atesora" (p. 625), mostrando entonces funciones del dinero como la captación de información y empleando de herramientsa a los precios y con esto termina siendo el camino del cálculo económico, como lo describió Mises: "aquello que permite el cálculo de los valores de mercado y entonces hace posible las elecciones individuales descentralizadas, estos argumentos permiten indagar sobre la hipótesis de neutralidad del dinero". Aparte, Mises ratifica la naturaleza dinámica del dinero desde sus inicios, como lo destaca en su libro "La acción humana", es ser cambiante puesto que

... al considerar de índole neutral, el dinero es tan erróneo como el creer en la plena estabilidad del poder adquisitivo del mismo. Una moneda, privada de la típica fuerza impulsora del dinero, contrariamente a lo que supone la gente, en modo alguno constituiría una valuta perfecta; antes, al contrario, dejaría de ser dinero (Von Mises, 1949, p. 626).

Dicho de otro modo, al crearle al dinero una característica de estabilidad se alteraría por completo su función, origen y razón de ser en las economías. Este autor también considera que los mecanismos de creación monetaria introducen perturbaciones permanentes en el funcionamiento de los mercados y según él, el dinero nunca es neutro.

Las funciones del dinero que conocemos en la actualidad no son del todo válidas, se reconoce al dinero como una unidad de cuenta, un método de intercambio y depósito de valor, de las anteriores, sólo una es la función del dinero, es un método de intercambio, las demás son características impuestas. Como lo menciona Von Mises (1949), son subsunciones de la función de intercambio y la demanda de todo medio de intercambio viene de ser resultante engendrada por las dos parciales demandas: de consumo y producción o medio intercambio aquí existe una uniforme proporcionalidad entre los precios y las variaciones cuantitativas del dinero.

Ahora bien, tomando en cuenta la hipótesis de neutralidad del dinero, hallaríamos 
un mercado convergente al mismo punto de equilibrio que hubiese sido de no haber existido cambios en la cantidad de dinero; por lo tanto, en a largo plazo no importa si el Banco Central sigue una regla, el dinero no generará afectaciones en el largo plazo y los precios son relativos, entonces el nivel de precios no importa porque la cantidad de dinero no es relevante para la estructura de equilibrio de mercado. Ahora bien, Von Mises (1949) enfatizó en que "la ausencia de acción no sólo es consecuencia del perfecto bienestar, sino también obligado corolario de la incapacidad de prosperar. Lo mismo puede significar desesperanza de la felicidad" (p. 626). Por parte de los Estados, entre más planes pretenden estructurar para la generación de un dinero neutro y estable resultan contradictorios por la misma naturaleza del dinero de ser un engendrador de cambio.

Ahora bien dentro de otra visión de los austriacos, el dinero se aleja mucho de ser neutro (para la teoría impuesta por Hayek, 1976), los economistas clásicos admiten la creación monetaria como la capacidad de estimular artificialmente la demanda y generando así, afectaciones sobre las condiciones reales de la producción; dentro de las ambiciones de Hayek se planteaba igualmente que el dinero implica distorsiones en la vida económica y en el ciclo económico, por ende, afirman que una moneda neutra no tiene directamente nada que ver con la estabilidad del nivel de los precios.

Por último, la neutralidad termina siendo un principio sin sentido y el más bajo del momento histórico en el que nos encontramos, donde se ven los diversos procesos de libre cambio, aumentos de globalización, y avances informático, entre otros; también se pone un poco del punto de regulación, ya que, dentro de la política monetaria de los Estados, están puestos sobre la mesa los intentos de neutralizar el medio de cambio, generando distorsiones económicas, tanto en los ciclos económicos, como en los niveles de precios. Apostar por la neutralidad del dinero terminará siendo una forma de generar una ruptura en la economía al abogar por la misma destrucción, puesto que se generarían procesos inflacionarios, llegando a tal punto que hasta a la economía más fuerte se afectaría.

\section{La propuesta de los austriacos de banca libre}

La propuesta austriaca se basa en el establecimiento de monedas privadas emitidas competitivamente, en lugar de mantener un número inmanejable de monedas, los mercados convergirían en una o solo un número limitado de normas monetarias, en las cuales se basarían las instituciones con base a su calificación de solidez. Las instituciones emitirían y regularían su moneda principalmente a través de préstamos y, en segundo lugar, a través de actividades de compra y venta de divisas. En otras palabras, crear un sistema de dinero de emisión privada en competencia pasiva, emitido por banqueros particulares respaldados por "cestas de mercancías" garantizando un valor mínimo a dicho dinero, y para ello es vital abolir el monopolio de emisión histórico en la economía para poder abrir las puertas en mayor medida al comercio, pero realizando limitaciones en las depresiones, ciclos de desempleo y procesos inflacionarios (Hayek, 1976)

La emisión libre existe desde el siglo XVII y consiste en dar a cada banco privado la capacidad de emitir sus propios billetes según el tamaño de sus activos, es decir, acorde a su capacidad para respaldarlos. Con el tiempo este sistema se fue abandonando debido a la inestabilidad monetaria que suponía y a la falta de 
transparencia de algunas entidades, sobreviviendo en el siglo XXI sólo en algunos casos aislados como Hong Kong.

Autores como Hayek (1976), son partidarios de la "libertad bancaria" y el "dinero libre" al existir restricciones por parte del Estado. El autor plantea una hipótesis en su libro "La desnacionalización del dinero", donde propone que si hubiese una sana moneda se operaría sin tener efecto en quien lo emita, ya que los recuentos históricos han mostrado como los países han usado las monedas para el sostenimiento de los mismo pero también para el aprovechamiento y caos en ciertas épocas, ya que la instauración del monopolio de emisión ha generado procesos de inflación, declives de la economía y se ha usado como un juguete de política gubernamental

Pese al respaldo de cada moneda por su país, el autor plantea la posibilidad de competencia de monedas, como ha ocurrido antes, sean los mismos usuarios quienes desplacen las monedas hasta llegar a transar con aquella de mayor confianza y beneficios les presente, es decir, si se derroca el monopolio de emisión y se les permite a las empresas privadas la emisión, se daría un proceso más eficiente que generaría competitividad.

El tema de la necesidad de que el Banco central sea quien regule y emita la moneda es una necesidad impuesta y que a través del tiempo ha perdurado, al ser el mismo Estado quien ha imposibilitado la competencia de monedas al considerar que traería fuertes afectaciones a la economía, además de bajar los ejes de poder en los cuales el sistema se desarrolla, existe manipulación, y para mantener el monopolio se argumenta lo poco confiables y no ser avaladas por los Bancos Centrales. Ahora bien, desde la perspectiva de la banca libre los Bancos centrales han limitado las acciones de las naciones con el beneficio particular de la emisión, al no permitir la competencia de la moneda, que ha impedido establecer el mejor método de transacciones con beneficios generales a las naciones.

El Estado ejerce monopolio sobre la unidad de cuenta, sin embargo, la emisión de dinero a través del sistema bancario no es tan fácil de controlar y puede generar ciertas afectaciones a las economías, y cabe resaltar la relación entre el proceso de regulación y la inflación. Como lo relata Hayek en su libro, los Estados se convirtieron en agentes totalmente inadecuados para esta tarea, especialmente tras observar como cada vez más y de forma más generalizada han abusado de su poder para defraudar a la gente, financiando sus propios déficits, generando inflaciones o devaluando su moneda (Hayek, 1976). Incluso apunta a que en ocasiones esa inflación ha sido generada por los gobiernos para su beneficio y resalta como, si los individuos comprendieran los costes que soporta en forma de inflación e inestabilidad por el hecho de utilizar un solo tipo de moneda en las transacciones y reflexionara sobre las ventajas de emplear varios, comprendería que esos costes son excesivos. Hayek (1976) resalta la importancia de privar al Estado de este privilegio, ya que la posibilidad de que el déficit estatal sea financiado mediante la emisión de dinero ha sido uno de los pilares de la expansión del poder de éste.

En cuanto a la competencia, el austriaco propone un sistema en el cual los bancos emiten de forma competitiva dinero privado, con el objetivo de imponer bajo este sistema monetario una fuerte disciplina a las instituciones financieras imposibilitando la emisión de dinero, sustancialmente menos seguro y útil que el dinero de cualquier otro banco, y da el poder a los usuarios de escoger el mejor medio 
de transacción y la misma competencia mostrará la combinación de mercancías que debe constituir en cualquier tiempo y lugar.

Caracterización de las monedas virtuales

Las monedas virtuales son hasta el momento un campo no tan explorado, pero sí que ha generado controversia por su origen, funcionamiento, y capacidad de almacenamiento de información, pero también por la alta volatilidad que la caracteriza. Las criptomonedas nacen en el año 20082009 con el denominado "bitcoin", donde se implementó la tecnología innovadora del blockchain y la criptografía. Adicionalmente se pretende proponer como las criptodivisas se convertirían en un método más común para las transacciones comerciales del día a día.

\section{Blockchain y criptografía}

El poder Estatal ha logrado parcializar la opinión de las personas, al plantear que las monedas nuevas como Bitcoin y criptomonedas son desconfiables, por los problemas centrales que plantean sus enemigos como son la alta volatilidad de la moneda y la incertidumbre que presentan. Sin embargo, para sus defensores son a la vez causa de la política de regulación de los Estados, ya que rezagan las posibilidades de competencia y la vuelven especulativa.

$\mathrm{El}$ sistema opuesto al presente régimen es el del curso voluntario o libre, donde es potestad de cada acreedor o vendedor aceptar, o no, determinado medio de intercambio como pago, lo que implica al individuo tener la libertad de escoger el dinero del emisor que considere más conveniente. Es una alternativa más segura al tener una tecnología que implementada permite generar realmente confidenciali- dad en sus transacciones. El blockchain y su criptografía intentan solucionar el problema del gasto doble, al ser intermediarios en las transacciones y además de esto, con la creación de libros contables públicos, descentralizados y distribuidos en cada nodo de la red es su mecanismo de funcionamiento y garantiza la seguridad de los datos.

Esta creación anónima tiene un sistema específico de trabajo en la tecnología del blockchain, y el análisis económico de este proceso no debe ser limitado, sino que debe tomar en cuenta todas las posibles estructuras, por ello el análisis comprende dos enfoques en los cuales se categoriza y caracteriza. Davidson et al (2018) consideran que es una realidad y una nueva forma de coordinación de las actividades económicas al tener una nueva forma como institución económica y la asemejan a una tecnología elemental del capitalismo de mercado moderno y al arte de gobernar.

En cuanto a la estrategia blockchain, se da a conocer un artículo importante de encadenamiento de bloques ligados al "bitcoin" para el 2008, el cual dio vía libre a una gran cantidad de noticias sobre el desarrollo de distintos sectores, donde se muestran las múltiples posibilidades para experimentar en el sector de la cooperación al desarrollo, generando un impacto transformador en el sector, siendo parte de la economía colaborativa; esta tecnología permite guardar una copia de los datos y detalles de cada transacción en ambos lados de la misma de manera en que los bloques de cada operación se enlazan como eslabones de una cadena con la seguridad que no podrán ser borrados o modificados. En todo sistema en el que haya algún tipo de intercambio, la cadena de bloques podrá mejorar su transparencia y transabilidad. 
La tecnología que implementa el blockchain tiene varios análisis, uno es la capacidad de ser un factor de aumento y el dos son las tecnologías sociales cuyos objetivos es solucionar el problema social y atender a los requisitos de simplicidad, bajo costo, fácil aplicabilidad (y replicabilidad) e impacto social comprobado. Como el blockchain puede facilitar los procesos comerciales y demás, pero su auge no ha sido tan pronunciado por que no existe una "legalidad" en las criptodivisas, esto limita su uso ya que la decisión de uso va relacionado a cuan adverso sea al riesgo de cada individuo al no contar con una garantía que permita confiar en otro medio de comparación.

Bajo la teoría económica del análisis de la "cadena de bloques", el tema se analiza desde dos enfoques, un enfoque neoclásico y un enfoque institucional, donde el primero lo define como la productividad de factores y el segundo hace referencia a las tecnologías sociales, es decir, un cambio institucional en la eficiencia. De manera más específica, en referencia al enfoque neoclásico se reconoce como un factor de aumento, un impulso al crecimiento con mejora en la eficiencia y reduce las ineficiencias, y el otro lo ve como una tecnología superior que da lugar a un sistema de pagos y el lente empresarial lo adopta por que produce ganancias marginales de eficiencia productiva.

En cuanto a la visión institucional mencionan varias variables como la incertidumbre, los activos y la frecuencia de relaciones, y aceptan que es más que una tecnología de coordinación económica, porque tiene la capacidad de disminuir los costos de transacción (por la eliminación de los intermediarios). Davidson et al (2018) se refieren a la existencia de la contribución al crecimiento en la forma de economización de los recursos escasos.
Aunque existen otros como Catalini \& Gans (2006), que consideran al blockchain afecta la economía bajo el lente cousiano y no schumpeteriano, considerandolo como perjudicial al perturbar las rentas económicas, por la eliminación de la intermediación. Dentro del enfoque neoclásico habría dos posiciones, una afín a las ideas y otra negativa a estas.

\section{Condiciones para su aceptabilidad}

Es claro que por la naturaleza de las criptomonedas o monedas digitales es alto su nivel de incertidumbre y por ende su uso va dirigido a los usuarios con mayor nivel de aversión al riesgo, sin embargo, en este apartado se pretende poner en conocimiento como se generaría aceptabilidad en los usuarios para convertirse en un medio transaccional de carácter normal o regular en la economía.

Si relacionamos el proceso de criptomonedas, blockchain con visiones como la de la escuela austriaca estaremos visualizando una nueva organización social y coherencia en los discursos teóricos de los austriacos, donde la estructura capitalista se vería afectada por su forma de funcionamiento e instauración en el mundo, la cual ha sido por medio de la intermediación y manejo de las monedas, limitando la libertad monetario de los usuarios. Esta nueva posibilidad se puede usar cuando la autora hace diversas distinciones sobre su forma de orientación y su implementación ratificando la posibilidad de moneda privada.

García-Arenas y Guasch (2018) realizan un proceso de análisis en su entorno y distinciones, subdividen en dos partes las monedas: moneda digital privada (MDP) y moneda digital respaldada (MDBC); si su característica es privada (como la naturaleza de la mundialmente conocida 
moneda bitcoin), la cual fue desarrollada bajo un código abierto, lo que significa que cualquier desarrollador puede contribuirla con el proyecto; en general es un proceso cooperativo donde se puede realizar la modificación de la fuente del programa sin restricciones de licencia (Nakamoto, 2018). Esta es una opción para generar la aceptabilidad de la moneda virtual, es decir, que el Banco Central respalde las transacciones, pero no que intervenga en el proceso para no perder la naturaleza que tiene, no obstante, permitiría tener un beneficio a las personas no tan adversas al riesgo que empiecen a "jugar" con esta moneda en el mercado.

También se logra identificar como en cuanto a las proyecciones de las criptomonedas se muestran complicaciones presentes en la moneda por como lo menciona el autor, las limitaciones sesgan su posibilidad de éxito. Por otro lado, según García-Arenas y Guasch (2018), en su artículo "El dinero digital en la economía del futuro: Nuevas posibilidades, nuevos retos" presentan la posibilidad de que este método que ha sido el hito del siglo XXI, sea extendido con éxito, pero garantizando su curso legal, generando una mayor opción de ampliación y cobertura en temas de uso y lograr su cometido de ser transable. En cuanto a la moneda MDBC, que cuenta con respaldo de la entidad institucional, se le convierte en la alternativa más viable, al contar con el proceso de reconocimiento, no de confianza, pero que permite dar un parte de tranquilidad a los usuarios para ser los encargados de decidir la mejor forma de intercambio.

En cuanto a la implementación se identifican dos posibles. La primera es la inserción disruptiva, que hace referencia a una innovación que ayuda a crear una nueva red de valor y eventualmente interrumpe el mercado actual en unos pocos años o décadas, desplazando una tecnología anterior y una segunda, la cual es la inserción al mercado de manera restringida, como es el caso contrario de los antes mencionados. El tema de implementación se da únicamente pensando en la posibilidad de la orientación de la moneda, como se menciona con anterioridad, al tener el respaldo o garantía del legislador. Claramente la evaluación de los costos y ventajas de las monedas digitales es vital, pero en un análisis podría mencionarse como son mayores los beneficios que los riesgos, del dinero del futuro. La tecnificación del mundo digital ha logrado reforzar el mecanismo de transacción de las monedas futuras, el método usado es el blockchain o lo que significa, "cadena de bloques", la cual es una base de datos distribuida y segura que mantiene en el anonimato las transacciones de sus usuarios; este ha causado gran impacto entre las generaciones puesto que existe una eliminación de intermediarios, en general realizando la descentralización de la gestión, generando a su vez una agilización de los sistemas efectivos de pago. Esta estrategia posee la acción de cooperación por ser esta base de datos distribuida y segura, empleada por una serie de mineros que trabajan para hacer de este una herramienta fiable, visto de una manera más sencilla se puede identificar como un gigantesco documento de cuentas, en el cual se presentan los registros enlazados y cifrados para proteger la seguridad y privacidad de las transacciones.

\section{Conclusiones}

La eliminación del monopolio instaurado por el Estado (gobierno) permitiría llevar a la economía a un curso estable de libertad bancaria. El protocolo de retroalimentación que sustituye a la supervisión, 
despliega un mercado similar al mecanismo (reputación y precio) para permitir la creación colaborativa y distribución del valor en redes de pares. El sistema se basa en un protocolo específico para habilitar redes de pares distribuidas para contribuir a una organización. A través del protocolo de retroalimentación basado en blockchain se puede coordinar indirectamente, explotando mutuamente su conocimiento especializado (Hayek, 1945). El monopolio gubernamental de la emisión del dinero generando un proceso de ineficiencia con relación a la calidad y el costo en el bienestar de los ciudadanos de hecho elimina el monopolio y permite incrementar el bienestar social, razón principal de la instauración mal infundada.

Llegar a la concepción de que el dinero privado es un gran aporte la alternativa de sistemas monetarios, permite la competencia, y realmente permite conocer no solo las preferencias de los usuarios y comportamiento de los mismos (legitimidad de entrada) sino también incluye la eficiencia de las metas al permitir dar este proceso, sin embargo, bajo el modelo económico en el cual vivimos se ve una posibilidad demasiado lejana en general que impide el funcionamiento de la economía en la imposibilidad de tener el sistema de desarrollo como un "mercado libre" por la estructura de precios que tambalea por factores como el empleo de este sistema.

La forma más plausible de tener retornos netos positivos en las economías es dejando actuar y permitir la competencia puesto que las acciones limitantes generan situaciones coyunturales mucho más fuertes de regular. Así que, el hecho de por lo menos admitir las criptomonedas como jugadores aceptados en la economía terminaría generando obstáculos a la criminalidad y decrecería la alta incertidumbre impuesta por el mismo gobierno obstruyendo el ingreso de personas no tan adversas al riesgo.

Como ya lo han mencionado referentes históricos como Mises, Hayek, Wicksell y los clásicos, el aspecto de neutralidad y el abogar por cumplirla en el dinero, no es el mejor plan a seguir, porque ya es bien conocido como el intento por los entes gubernamentales terminaría en afectaciones mayores a las economías. La recomendación es dejar ser al dinero, es decir, permitirle que sea libre y que no se le trunque su naturaleza.

Dentro del análisis realizado de manera documental se puede asegurar: el BITCOIN si es una materialización de la idea austriaca, ya que cumple con todos los aspectos mencionados por Hayek y la escuela austriaca, y cumple con ser un medio descentralizado que brinda la seguridad suficiente a los usuarios, y no está ligado a ningún poder estratégico estatal y su regulación no es real, adicionalmente tiene de manera implícita su forma de competencia con demás monedas de carácter digital y con el papel moneda. El BITCOIN, dinero fiduciario se asemeja mucho al oro puesto que no es responsabilidad de nadie.

\section{REFERENCIAS}

Catalini, C. \& S. Gans, J. (2016). Some Simple Economics of the Blockchain. MIT Sloan Research Paper No. 5191-16. [Online]. http://dx.doi. org/10.2139/ssrn.2874598

Davidson, S., De Filippi, P. \& Potts, J. (2018). Blockchains and the economic institutions of capitalism. Journal of Institutional Economics, 14(4). 639-658. http://dx.doi. org/10.1017/S1744137417000200 
Friedman, M. [Reddit] (19 de Febrero de 1999). Professor Milton Friedman predicts Crypto in 1999 . [Archivo de video]. Recuperado de www.reddit.com/r/CryptoCurrency/ comments/8bvg4e/professor_milton_ friedman_predicts_crypto_in_1999/

García-Arenas, J. \& Guasch, M. (2018). El dinero digital en la economía del futuro: nuevas posibilidades, nuevos retos. CaixaBank Research, 423[Online]. 37-39. Recuperado de http:// www.caixabankresearch.com/sites/ default/files/documents/im_1805_3739_dossier_3_es_0.pdf

Hayek, F. (1976). La desnacionalización del dinero: Un análisis de la teoría y práctica de las monedas concurrentes. Londres: Institute of Economic Affairs.

Hayek, F. (1945). El uso del conocimiento en la sociedad. American Economic Review, 25(1). 519-530.

Nakamoto, S. (2009). Bitcoin: Un Sistema de Efectivo Electrónico Usuario-aUsuario. Hawthorne: bnpublishing. com. Recuperado de https://bitcoin. org/files/bitcoin-paper/bitcoin_es.pdf

Von Mises, L. (1949). K! Cambio indirecto. En, L, Von Mises, La acción humana, tratado de economía (4 ed.). 595-695 New Haven: Yale University Press.

\section{Biodata}

Daniela Alejandra Montaña Rubiano es estudiante de séptimo semestre en Economía de la Universidad Colegio Mayor de Cundinamarca (Colombia). https://orcid. org/0000-0002-5676-2798 